\title{
A POSSIBILIDADE DE CONTRIBUIÇÃO DA ACUPUNTURA NO ENSINO DA SIMULAÇÃO CLÍNICA EM ENFERMAGEMª
}

\author{
M ateus Casanova dos SAN T OS ${ }^{b}, M^{2}$ aria Cecília L orea LEITEcc, Rita M aria HECK ${ }^{d}$
}

\section{RESUMO}

0 presente estudo situa a discussão sobre a possibilidade emergente da inserção dos conhecimentos oriundos da acupuntura como contribuição para as práticas pedagógicas do ensino clínico simulado da graduação em enfer magem. 0 trabal ho reflexivo emergiu como um recorte do projeto de dissertação "E studo da avaliação no disparador de aprendizagem simulação - L aboratório M orfofuncional/ F aculdade de E nfer magem/ U niversidade Federal de Pelotas". A relação integral entre o homem e a natureza desenvolvida na acupuntura emerge neste estudo como discussões e como uma sugestão potencial enquanto ferramenta pedagógica para a simulação clínica da enfer magem. N esta reflexão, certifica-se que há a necessidade de desenvolver este recurso pedagógico com vistas a ampliar o ensino da simulação clínica em enfer magem.

Descritores: E ducação em enfermagem. Simulação. A cupuntura.

\section{RESUMEN}

E ste estudio sitúa el debate surgido sobre la posibilidad de integración de los conocimientos de la acupuntura como una contribución a las prácticas pedagógicas de la educación si mulada clínica dela enseñanza de pregrado. E I trabajo de reflexión surgió como una aproximación al proyecto de tesis "E studio sobre la evaluación del aprendizaje de simulación de activación - morfo- L ab/ E scuela deE nfermería/ U niversidad F ederal deP elotas". L a relación integral entreel hombrey la natural eza desar rollada en la acupuntura emerge como una suger encia de los debates y el potencial como una her ramienta pedagógica para la simulación de la enfer mería clínica. E n esta reflexión, se certifica que hay una necesidad de desarrollar este recurso pedagógico con el fin de ampliar la enseñanza de la simulación clínica en enfermería.

Descriptores:Simulación. E ducación en enfermería. A cupuntura.

Título: L a posibilidad de contribución de acupuntura en la enseñanza de la simulación clínica en enfer mería.

\section{ABSTRACT}

This study situates theemerging discussion about the possi bility of integration of know ledgefrom acupunctureas a contribution to the pedagogical practices of simulated clinical education of undergraduatenursing education. The reflective w ork emerged as an approach to the dissertation project "Study on the eval uation of trigger learning simulation - M or phofunctional L ab/ College of N ursing/U niversidade F ederal de Pelotas". The integral relationship between man and nature developed in acupuncture emerges as a suggestion of discussions and a potential pedagogical tool for the clinical simulation in nursing. In this reflection, results provee that there is a need to develop this educational resource aimed at expanding the teaching of clinical simulation in nursing.

Descriptors: Simulation. N ursing, education. A cupuncture.

Title: Possible Contributions of A cupuncture in the Teaching of Clinical Simulation in N ursing.

\footnotetext{
a Recorte do projeto de pesquisa de dissertação intitulado Estudo da avaliação no disparador de aprendizagem Simulação do Laboratório M orfofuncional da Faculdade de E nfermagem da U niversidade Federal de Pelotas (U F Pel).

b M estre em E nfermagem, E special ista em A cupuntura e E letroacupuntura com orientação antroposófica, Professor A ssistente do Instituto de Biologia da U F Pel, D ocente do Curso de Enfermagem da Faculdade A nhanguera E ducacional, Pelotas, Rio G rande do Sul, Brasil.

' $D$ outora em E ducação, Professora A djunta da F aculdade de E ducação e do Programa de Pós-G raduação em E ducação da U F Pel, Pelotas, Rio Grande do Sul, Brasil.

d D outora em Enfermagem, Professora A djunta da Faculdade de Enfermagem e do Programa de Pós-G raduação em Enfermagem da UF Pel, Pelotas, Rio Grande do Sul, Brasil.
} 


\section{CONSIDERAÇÕESINICIAIS}

As terapias complementares em saúde, aprovadas no Brasil em 2006, estão sendo observadas e desenvolvidas no Sistema Ú nico em Saúde (SU S), através de pontos sentinela de atendimento espaIhados no território nacional( ${ }^{(1)}$. $\mathrm{Na}$ Enfermagem gaúcha, por exemplo, destaca-se os atendimentos em terapias complementares em saúde realizados por enfermeiros da sede da Associação Brasileira de T er apias $\mathrm{N}$ aturais em Saúde (A BRAT E N ), Porto A legre, R io $\mathrm{G}$ rande do Sul, com o apoio ConseIho Regional de Enfermagem do Rio Grande do Sul (COREN/RS).

Como há inúmeras discussões e reflexões desta temática que repercutem no ensino, na assistência e no trabalho interdisciplinar da Enfermagem e tendo em vista a necessidade da participação da profissão na regulamentação legal para a atividade de acupuntura a fim de estabelecer os limites e a abrangência desta prática ${ }^{(2)}$, pretendeu-se desenvolver esta reflexão a cerca das contribuições da acupuntura, enquanto terapia complementar em saúde e prática oriental em saúde, no ensino clínico do laboratório de simulação da F aculdade de E nfermagem (FE n) da U niversidadeF ederal de Pelotas (UF Pel).

A FEn/UFPel percorre um momento especial de reestruturação curricular planejado desde 0 ano de 2002 e iniciado em 2009. A proposta curricular está fundamentada por habilidades e competências ${ }^{(3)}$, metodologia problematizadora ${ }^{(4)}$, visando a for mação de um aluno pró-ativo e considerando o SU S como espaço de formação profissional do enfermeiro. A indicação do Projeto Político-Pedagógico da Faculdade de Enfermagem/ U FPel está total mente contemplada na organização curricular por A prendizagem Baseada em Problemas (ABP) e, neste movimento, o ensino-aprendizagem é facilitado pelo professor que estimula 0 processo crítico-reflexivo ${ }^{(5)}$.

$\mathrm{N}$ esta perspectiva curricular, o disparador de aprendizagem Simulação é caracterizado como um espaço protegido que simula cenários da prática de cuidados à saúde para pequenos grupos de acadêmicos de E nfermagem, onde os estudantes realizam atendimentos em pacientes simulados, realizam procedimentos em manequins e/ ou bonecos, entre outras atividades pedagógicas problematizadoras e clínicas ${ }^{(6)}$.

0 objetivo deste estudo teórico-reflexivo ésituar possibilidades de diálogo entre a acupuntura e o ensino na prática da simulação junto ao Laboratório M orfofuncional da FEn/ UFPel, aproximando o processo ensino-aprendizagem às necessidades do Sistema Ú nico de Saúde e da Política Nacional de Práticas Integrativas e Complementares em Saúde (PN PIC), sobretudo, embasados nas D iretrizes Curriculares para a E nfermagem ${ }^{(7)}$.

As diretrizes permitiram que os currículos construam o perfil acadêmico e profissional com competências, habilidades e conteúdos conforme as perspectivas nacionais e internacionais, capacitando para atuar no SU $S^{(8)}$.

Este trabalho emergiu como um recorte do projeto de pesquisa de dissertação, intitulado "E studo da avaliação no disparador de aprendizagem simulação - Laboratório M orfofuncional/ F aculdade de Enfermagem/ UF Pel" (Comitê de Ética em Pesquisa da F En-U F Pel, Parecer 60/2010). A discussão sobre a possibilidade da inserção da acupuntura como forma de contribuição para o ensino clínico simulado em enfermagem é uma ideia que emergiu a partir das vivências profissionais do autor nas terapias complementares em saúde e, sobretudo, durante a observação de campo da pesquisa no disparador de aprendizagem Simulação da FEn/ U F Pel. 0 amadurecimento das reflexões também tiver am a contribuição das discussões geradas na FEn/ U F Pel através do projeto "Plantas bioativas de uso humano por famílias de agricultores de base ecológica da região sul do RS", desenvolvido em parceria com a E mbrapa Clima Temperado.

\section{ENSINO DA PRÁTICA DA SIMULAÇÃO NO LABORATÓRIO MORFOFUNCIONAL}

Frente a essa nova realidade curricular na FE n/ U F Pel, no dia 06 de julho de 2009, inaugurou nas dependências da instituição o L aboratório Prático M orfofuncional, assegurando, ao acadêmico, espaço potencial próprio para o aperfeiçoamento profissional exigido no novo currículo. Esse espaço de ensino é utilizado sobremodo no disparador de aprendizagem denominado Simulação.

A prática de simulação pertence à terceira área do currículo e é considerado um disparador de aprendizagem junto à pedagogia baseada em problemas ${ }^{(9)}$. Como esta prática revela-se importante para 0 ensino em enfermagem ${ }^{(10,11)}$, no currículo da FEn/UFPel ela pretende desenvolver as capaci- 
dades necessárias ao domínio da competência nas áreas de saúde, de gestão e sistematização da assistência ${ }^{(9)}$.

Enquanto no primeiro ano, a simulação trafega pelos conhecimentos semiológicos e semiotécnicos da enfermagem, no segundo ano, infere sobremodo as práticas e os procedimentos da enfermagem hospitalar. Portanto, o conhecimento morfofuncional clínico é iniciado no primeiro ano do novo currículo e é uma necessidade que se torna inerente na formação das habilidades dos profissionais em saúde ${ }^{(12)}$.

\section{A POSSIBILIDADE DE CONTRIBUIÇÃO DA ACUPUNTURA NO ENSINO DA SIMU- LAÇÃO CLÍNICA EM ENFERMAGEM}

A acupuntura chinesa como uma cultura milenar é baseada na filosofia taoísta e nela o universo e o ser humano estão submetidos às mesmas influências da natureza, sendo partes integrantes do universo como um todo. Esta concepção está apoiada em três pilares básicos: a teoria do Yang/Y in, dos Cinco M ovimentos (Terra, Água, Ar, Fogo e $M$ etal) e dos Zang $F u$ (Órgãos e V ísceras) (13,14).

0 plano morfofuncional do organismo humano, a partir da tradição oriental da acupuntura, é permeado de locais superficiais e profundos onde os meridianos e canais orgânicos percor rem e possuem alta condutividade de potencial elétrico ${ }^{(14)}$. $N$ estas regiões transitam o Q i carreado de informações neuroquímicas específicas e com al to poder de auto-organização quando devidamente acionados em um circuito ter apêutico(13,14).

$\mathrm{N}$ a prática da simulação do segundo semestre do primeiro ciclo da graduação, o acadêmico perpassa a semiologia e semiotécnica sistêmica corporal, desenvolvendo, respectivamente, o estudo das medidas antropométricas; da pele, cabelo e unhas; da cabeça e pescoço; dos ol hos, orel has, nariz e garganta; do tórax e pulmão; do coração; dos vasos sanguíneos e sistema linfático; do abdome; da genitália feminina e mamas; da genitália masculina e próstata; do ânus e reto; do sistema músculo-esquelético; e, do sistema neurológico. Observou-se que estas simulações reforçam a integração do conteúdo da anatomia humana e da fisiologia humana e, sob uma análise baseada em Bernstein ${ }^{(15)}$, fortalecem a classificação e 0 enquadramento do discurso pedagógico neste espaço micro de ensino através da interdisciplinaridade, da proposta metodológica ativa e do pensamento crítico-reflexivo por parte do adquirente.

N este ínterim, a visão integral homem-natureza desenvolvida na acupuntura ${ }^{(13)}$, operacionalizada enquanto forma terapêutica através da estimulação transcutânea por meio dos meridianos corporais e, inclusive, na racional idade diagnóstica global desta ciência milenar, emerge como discussão e como uma sugestão potencial enquanto ferramenta pedagógica do discurso instrucional para o espaço da simulação da enfermagem quando se simula o ensino clínico dos sistemas corporais humanos.

Neste movimento, acredita-se que 0 uso das maquetes e manequins do laboratório, o teatro clínico simulado entre os graduandos e as técnicas semiotécnicas desenvolvidas junto à visão integral do corpo humano através da acupuntura sejam potencial mente benéficas para o aprendizado morfofuncional, interdisciplinar e clínico no laboratório da simulação.

D esta forma, entende-se que o ensino da dinâmica relação morfofuncional humana pode ser mais integrada e vivificada pelo adquirente na sua prática clínica simulada, com vistas a aperfeiçoar, no decorrer da formação profissional, a autoconfiança e a percepção clínica morfofuncional durante 0 atendimento real ao paciente.

\section{CONSIDERAÇÕES FINAIS}

0 estudo exposto é considerado uma reflexão ousada e pretensiosa situando uma entre tantas possibilidades de diálogo entre 0 ensino de Enfer magem, a acupuntura, a P N PIC e o SU S. D esta forma, pretendeu-se dar início às discussões das práticas pedagógicas inovadoras e ativas das simulações morfofuncionais e clínicas em enfermagem junto a referencias complementares em saúde, neste caso, a acupuntura. A partir desta reflexão, certifica-se que há a necessidade de desenvolver este recurso pedagógico com vistas a ampliar o ensino da simulação clínica na enfer magem.

\section{REFERÊNCIAS}

1 M inistério da Saúde (BR), Secretaria de Atenção à Saúde, D epartamento de A tenção Básica. Política N acional de $P$ ráticas I nteg rativas e Complementares no SUS - PN PIC-SU S. Brasília (D F ); 2006. 
2 Kur ebayashi LF S, Oguisso T, F reitas G F. A cupuntura na enfer magem brasileira: dimensão ético-legal. Acta Paul Enferm. 2009;22(2):210-2.

3 Fernandes D. Avaliação das aprendizagens: desafios às teorias, práticas e políticas. L isboa: T exto; 2005.

4 G omes R, Brino RF, A quilante AG, Avó L RS. A prendizagem Baseada em Problemas na formação médica e o currículo tradicional de $M$ edicina: uma revisão bibliográfica. Rev Bras Educ Med. 2009;33(3):44451.

5 Semim GM, Souza M CBM , Corrêa AK. Professor como facilitador do processo ensino-aprendizagem: visão de estudantes de enfermagem. Rev G aúcha Enferm. 2009;30(3):484-91.

$6 \mathrm{~N}$ eil J. Simulation in nursing education. Periop N urs Clin. 2009;4:97-112.

7 M inistério da E ducação (BR), Conselho $\mathrm{N}$ acional de $E$ ducação. Resolução no 3, de 07 de novembro de 2001: institui Diretrizes Curriculares Nacionais do Curso de Graduação em Enfermagem. Brasília (DF); 2001 [ citado 2010 maio 02]. Disponível em: http:// portal.mec.gov.br/ cne/ arquivos/ pdf/ CE S 03.pdf.

8 Oliveira RA, Ciampone M HT. A univer sidade como espaço promotor de qual idade de vida: vivências e expressões dos al unos de enfer magem. T exto Contexto Enferm. 2006;15(2):254-61.
9 Souza AF, Jardim VM R, Coimbra VCC. A experiência de construção e implementação de um currículo de enfer magem orientado a partir do Sistema ú nico de Saúde [ Internet] . In: 20 Seminário $\mathrm{N}$ acional de D iretrizes de Enfermagem na A tenção Básica em Saúde; 2009 ago 20-22; Recife, Brasil. Recife: ABEn-PE; 2009 [ citado 2009 dez 05]. Disponível em: http:/ / www.abeneventos.com.br/SE NABS/ cd_anais.

10 Godson NR, W ilson A, Goodman M. Evaluating student nurse learning in the clinical skills laboratory. Brit J N urs. 2007;16(15):942-5.

11 Prion S. A practical framework for evaluating the impact of clinical simulation experiences in prelicensure nursing education. Clin Simul N urs. 2008; 4:69-78.

12 Z aid H, W ard D, Sammann A, T endick F, T opp KS, $M$ aa J. Integrating surgical skills education into the anatomy laboratory. J Surg Research. 2010;158(1): 36- 42.

13 Yamamura Y. A cupuntura tradicional: a arte de inserir. São Paulo: Roca; 2001.

14 Leake R, Broderick JE. T reatment efficacy of acupuncture: a review of the research literature. Integr M ed. 1998;1(3):107-15.

15 Bernstein B. Pedagogía, control simbólico e identidad: teoría, investigación y crítica. M adrid: M orata; 1996.

Recebido em: 20/ 05/ 2010

A provado em: 06/ 01/ 2011

\author{
Endereço do autor / Dirección del autor / \\ Author's address: \\ $M$ ateus Casanova dos Santos \\ Rua D r. Fer reira Soares, 244 \\ 96020-160, Pelotas, RS \\ E-mail: mateuscasanova@ig.com.br
}

